\title{
(2) MiR-3150b-3p inhibits the proliferation and OPEN ACCESS invasion of cervical cancer cells by targeting TNFRSF11a
}

\author{
Zhijuan Yu, ${ }^{1}$ Liguo Wang $\odot,{ }^{2}$ Xiujuan Li ${ }^{1}$
}

Additional material is published online only. To view, please visit the journal online (http://dx.doi.org/10. 1136/jim-2020-001284).

${ }^{1}$ Department of Obstetrics and Gynecology, Chifeng Municipal Hospital, Chifeng, China

${ }^{2}$ Department of Surgical Oncology, Chifeng Municipal Hospital, Chifeng, Inner Mongolia, China

\section{Correspondence to} Dr Liguo Wang, Department of Surgical Oncology, Chifeng Municipal Hospital, Chifeng 024000, Inner Mongolia, China; liguowang_12@163.com

Accepted 26 May 2020 Published Online First 2 July 2020

\section{Check for updates}

(C) American Federation for Medical Research 2020. Re-use permitted under CC BY-NC. No commercial re-use. Published by BMJ.

To cite: Yu Z, Wang L, Li X.J Investig Med 2020;68:1166-1170.

\begin{abstract}
The objective of this study was to determine the role of miR-3150b-3p in the cervical cancer (CC) progression. Real-time PCR and western blot analysis were conducted to test the expression of miR-3150b-3p, TNFRSF11a and p38 mitogenactivated protein kinase (MAPK) signaling pathway. The interaction between miR-3150b-3p and TNFRSF11a was verified by luciferase assay. Cell proliferation, migration and invasion were determined by CCK-8, wound healing and Transwell assays. In this study, we showed that miR-3150b-3p was significantly downregulated in CC cell lines. Additionally, miR-3150b-3p markedly attenuated the proliferation, migration and invasion of HeLa and SiHa cells. Moreover, we identified TNFRSF11a to be a novel target of miR-3150b-3p in CC cells. Enforced expression of TNFRSF11a abolished the antitumor effect of miR-3150b-3p. Besides, miR-3150b-3p was involved in the regulation of the p38 MAPK signaling pathway. In conclusion, our data suggested that miR-3150b-3p directly targets TNFRSF11a to inactivate the p38 MAPK signaling pathway, thus implicating miR-3150b-3p in the regulation of CC cell growth.
\end{abstract}

\section{INTRODUCTION}

By 2012, it is estimated that 528000 cases of cervical cancer (CC) had been diagnosed, of which 266000 died from CC worldwide. ${ }^{1}$ Currently, the treatment methods for CC mainly include surgery, radiotherapy and chemotherapy. ${ }^{2}$ However, in clinical practices, most patients with metastatic tumors have poor prognosis, resulting in unsatisfactory outcomes and even tumor recurrence. ${ }^{3}$

TNFRSF11a emerges as a pivotal regulator of cell differentiation, proliferation and survival. ${ }^{4}$ Furthermore, TNFRSF11a is identified as an inducement to activate dendritic cells and may also be crucial for the maintenance of immune tolerance. ${ }^{5}$ TNFRSF11a inhibits the motility and migration of breast cancer cells. ${ }^{6}$ Meanwhile, TNFRSF11a has been shown to promote the proliferation of CC cells, and inhibit apoptosis, suggesting that TNFRSF11a might be a possible target for CC therapy. ${ }^{7}$ Recently, TNFRSF11a was indicated to promote CC cell proliferation, migration and invasiveness via

\section{Significance of this study}

What is already known about this subject?

- MiR-3150b-3p acts as a tumor suppressor in colorectal cancer.

- TNFRSF11a promotes the proliferation of cervical cancer cells, and inhibits apoptosis.

- TNFRSF11a activities p38 mitogenactivated protein kinase (MAPK) signaling pathway in cervical cancer.

What are the new findings?

- MiR-3150b-3p is downregulated in cervical cancer cells.

- MiR-3150b-3p alleviates cervical cancer cell growth via decreasing TNFRSF11a expression.

- MiR-3150b-3p ameliorates cervical cancer tumorigenesis by targeting TNFRSF11 a via inhibiting p38 MAPK signaling pathway.

How might these results change the focus of research or clinical practice?

- MiR-3150b-3p might be a valuable target for developing therapeutic strategy against cervical cancer.

\section{Highlights}

- MiR-3150b-3p is significantly downregulated in cervical cancer cell lines.

- MiR-3150b-3p attenuates cervical cancer cell proliferation, migration and invasion.

- miR-3150b-3p acts as a tumor suppressor in cervical cancer by targeting TNFRSF11a via the inactivation of p38 MAPK signaling pathway.

the activation of $\mathrm{p} 38$ mitogen-activated protein kinase (MAPK) signaling pathway. ${ }^{8}$

MiRNAs are a class of endogenous noncoding RNAs, which negatively regulate gene expression by translational inhibition or degradation of target mRNAs. ${ }^{9}$ A growing body of evidence suggests that the abnormal expression of miRNAs play momentous roles in the tumorigenesis, including CC. ${ }^{10-12}$ In this study, using bioinformatics analysis (http:// www.targetscan.org/), we found that miR$3150 \mathrm{~b}-3 \mathrm{p}$ might bind to TNFRSF11a three prime untranslated region (3'-UTR) to inhibit 
the expression of TNFRSF11a. Based on the abovementioned findings, we thus speculated that miR-3150b-3p via targeting TNFRSF11a inhibited CC development by mediating the p38 MAPK signaling pathway. Our findings will provide a novel mechanistic insight into a critical role of miR-3150b-3p in CC, and shed new light on miRNAdirected diagnostics and therapeutics in CC.

\section{MATERIALS AND METHODS \\ Cell culture}

An immortalized human cervical epithelial cell line HCerEpic and human CC cell lines (C33A, HeLa, CaSki and $\mathrm{SiHa}$ ), which were all obtained from American Type Culture Collection (Manassas, Virginia, USA), were incubated in antibiotics-free Dulbecco's Modified Eagle Medium (DMEM.

\section{Cell transfection}

$\mathrm{HeLa}$ and SiHa cells were transfected with indicated plasmids $(50 \mathrm{nM}$ ) using Lipofectamine 2000 (Life Technologies, Carlsbad, California, USA) according to the manufacturer's protocol.

\section{Luciferase reporter assay}

The fragments of the $3^{\prime}$-UTR of TNFRSF11a containing the predicted miR-3150b-3p binding sites were synthesized and cloned into the pmirGLO vectors (Promega, Madison, Wisconsin, USA) to obtain the reporter vector for TNFRSF11a 3'-UTR-wild type (WT). The corresponding 3'-UTR-mutated-type (MUT) was synthesized by GenePharma Corporation (Shanghai, China). These reporter plasmids were transfected into HEK293T cells. After 48 hours of transfection, cells were collected to test the luciferase activity.

\section{CCK-8 assay}

Cells were seeded in 96-well plate at a density of $1.0 \times 10^{4}$ cells/well and incubated at $37^{\circ} \mathrm{C}$ at $24,48,72$ and 96 hours after transfection. Then, cells were treated with $10 \mu \mathrm{L}$ CCK-8 reagent (Beyotime, Beijing, China) at $37^{\circ} \mathrm{C}$ for additional 2 hours. The optical density was measured at 450 nm.

\section{Migration assay}

Transfected cells were scratched with a $10 \mu \mathrm{L}$ pipette tip, washed with phosphate-buffered saline and re-suspended in culture medium for 24 hours at $37^{\circ} \mathrm{C}$. Representative images after wounding were captured with a light microscope (Leica Microsystems, Wetzlar, Germany).

\section{Transwell assay}

Cells resuspended in serum-free medium were added to the upper chamber. DMEM containing 10\% fetal bovine serum as a chemoattractant was added to the bottom chamber. After 24 hours, these cells failing to invade were removed from the upper part of the filters by scrubbing with a cotton swab. And the membrane was stained with $0.5 \%$ crystal violet for $10 \mathrm{~min}$, and counted under a light microscope (Leica Microsystems) at $200 \times$ magnification from 10 different fields of each filter.

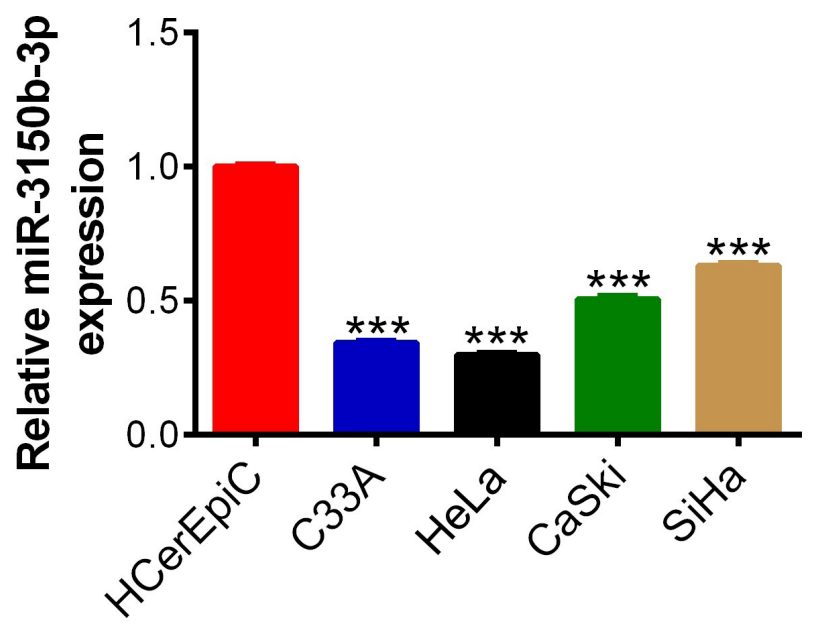

Figure 1 MiR-3150b-3p was downregulated in cervical cancer (CC) cells. The expression levels of miR-3150b-3p measured in four human CC cell lines (C33A, HeLa, CaSki and SiHa) and normal cervical epithelial cell line HCerEpic by real-time PCR method. Data were shown as mean \pm SD. Each experiment was conducted independently for three times. ${ }^{* * *} \mathrm{P}<0.001$ vs HCerEpic cells.

\section{RNA isolation, reverse transcription and quantitative real-time PCR}

Total RNA was isolated and subsequently reversely transcribed into complementary DNA using a reverse transcription kit (Takara, Shanghai, China). The mRNA expressions were determined by real-time PCR.

\section{Western blot analysis}

Total proteins were transferred to polyvinylidene fluoride membrane (Millipore, Bedford, Massachusetts, USA). After blocked with $5 \%$ skimmed milk at $4^{\circ} \mathrm{C}$ overnight, proteins were incubated with primary and secondary antibodies (Cell Signaling Technology, Boston, Massachusetts, USA) for 2 hours at $37^{\circ} \mathrm{C}$. The target proteins were visualized using ECL system (7Sea PharmTech, Shanghai, China).

\section{Statistical analysis}

Data were expressed as mean \pm SD. Statistical significance among groups was compared using one-way analysis of variance test.

\section{RESULTS}

\section{MiR-3150b-3p is downregulated in CC cells}

The results of real-time PCR analysis demonstrated that, compared with normal cervical epithelial cells HCerEpic, the expression levels of miR-3150b-3p were significantly lower in human CC cells (C33A, HeLa, CaSki and SiHa) (figure 1).

\section{MiR-3150b-3p inhibits CC cell viability}

The transfection efficiency of miR-3150b-3p overexpression or knockdown was confirmed by real-time PCR (figure 2A). As shown in figure $2 \mathrm{~B}$, overexpression of miR$3150 \mathrm{~b}-3 \mathrm{p}$ significantly suppressed cell viabilities of $\mathrm{HeLa}$ cells. However, downregulation of miR-3150b-3p remarkably increased cell proliferation abilities of SiHa cells. 
A

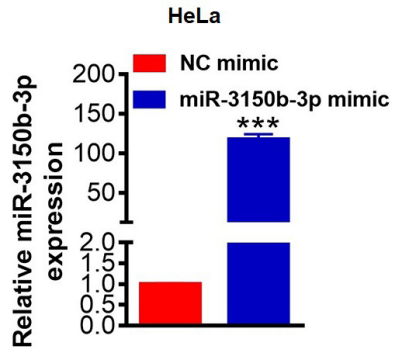

HeLa

B

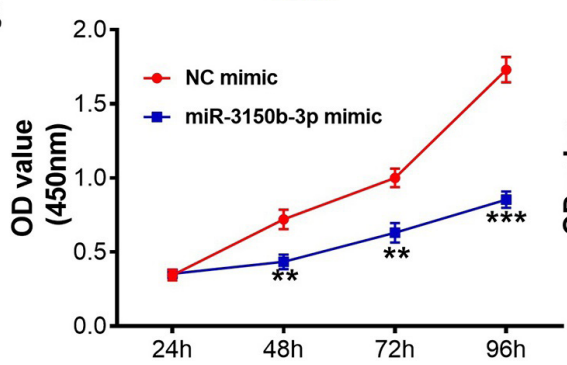

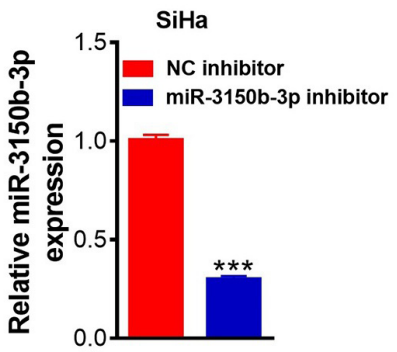

$\mathrm{SiHa}$

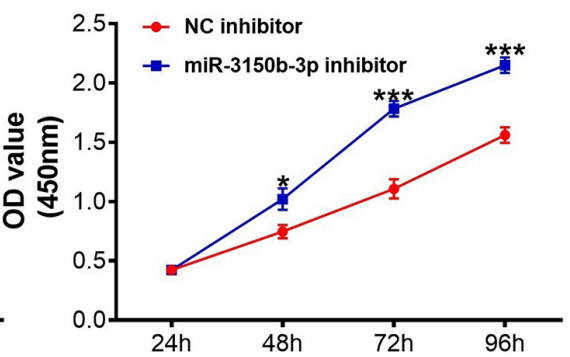

Figure 2 MiR-3150b-3p inhibited cervical cancer (CC) cell viability. The expression levels of miR-3150b-3p (A) and CCK-8 cell viability assay (B) in HeLa or SiHa cells with miR-3150b-3p mimic or inhibitor transfection, respectively. Data were shown as mean \pm SD. Each experiment was conducted independently for three times. ${ }^{*} \mathrm{P}<0.05,{ }^{* *} \mathrm{p}<0.01,{ }^{* * *} \mathrm{p}<0.001$ vs mimic/inhibitor NC group.

\section{MiR-3150b-3p suppresses migratory and invasive behaviors of CC cells}

The migratory abilities of HeLa cells were remarkably decreased following miR-3150b-3p knockdown, whereas,
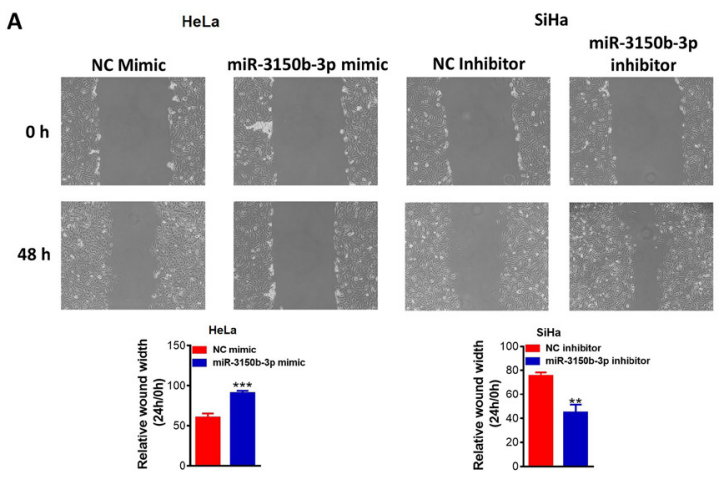

B

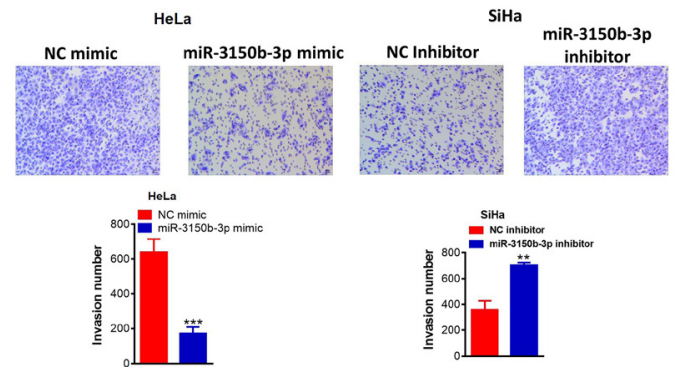

Figure 3 MiR-3150b-3p suppressed migratory and invasive behaviors of cervical cancer (CC) cells. Cell migration (A) and invasion (B) abilities of HeLa or SiHa cells with miR-3150b-3p mimic or inhibitor transfection were determined by wound-healing and Transwell invasion assays. Data were shown as mean \pm SD. Each experiment was conducted independently for three times. ${ }^{* *} \mathrm{P}<0.01,{ }^{* * *} \mathrm{p}<0.001$ vs mimic/inhibitor NC group. there was a remarkable increase in cell migration of $\mathrm{SiHa}$ cells with miR-3150b-3p overexpression (figure 3A). Furthermore, invasive abilities of Hela cells were significantly reduced with miR-3150b-3p mimic transfection, whereas, miR-3150b-3p inhibitor exhibited a reverse effect (figure 3B).

\section{MiR-3150b-3p targets TNFRSF11a in CC cells}

TNFRSF11a was a potential target gene of miR-3150b-3p (figure 4A). To verify whether TUSC5 was a direct target of miR-3150b-3p, we employed a dual-luciferase reporter system, showing that ectopic expression of miR-3150b-3p decreased the wild-type TNFRSF11a-3'-UTR luciferase activity but not that of its mutant in 3'-UTR luciferase assays. TNFRSF11a expression at both the mRNA and protein levels was significantly downregulated in HeLa cells after transfection with miR-3150b-3p mimic compared with these levels in the mimic NC group, while miR$3150 \mathrm{~b}-3 \mathrm{p}$ downregulation in SiHa cells led to an opposite effects (figure 4B-C).

\section{MiR-3150b-3p alleviates CC cell proliferation, migration} and invasion via targeting TNFRSF11a

Enforced expression of TNFRSF11a abrogated the inhibitory effects of miR-3150b-3p overexpression on the proliferation (figure 5A), migration (figure 5B) and invasion (figure 5C) in HeLa cells.

\section{MiR-3150b-3p ameliorates CC progression via targeting} TNFRSF11a by inactivating p38 MAPK signaling pathway The protein levels of TNFRSF11a, p-p38, proliferationassociated proteins (survivin and c-myc) and invasionrelated proteins (matrix metalloproteinase (MMP)-2, MMP-9) were downregulated by miR-3150b-3p overexpression in HeLa cells, without a change in total p38 
A

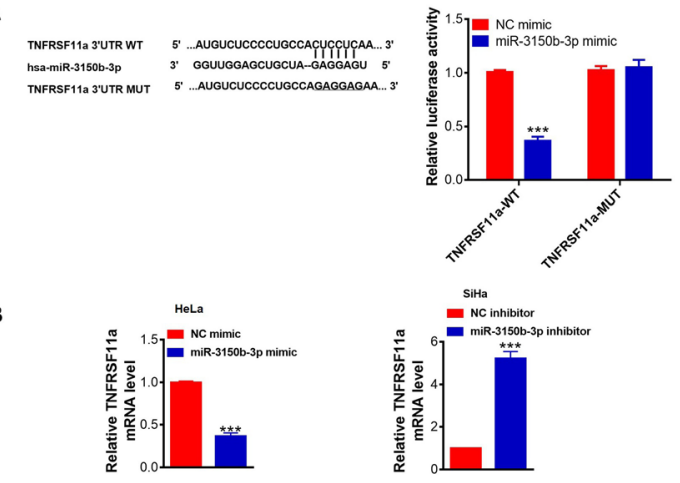

c

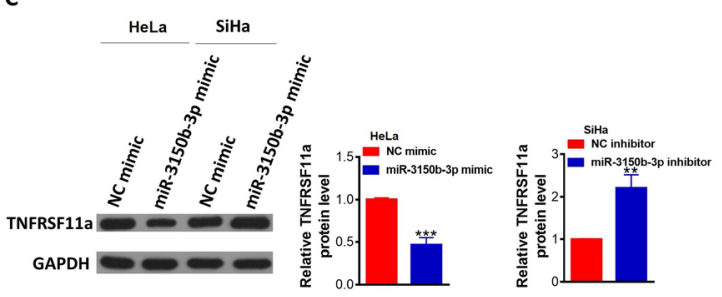

Figure 4 MiR-3150b-3p directly targeted TNFRSF11a in cervical cancer (CC) cells. The potential binding site of TNFRSF11a three prime untranslated region (3'-UTR) for miR-3150b-3p was predicted by bioinformatic analysis (TargetScan); luciferase activities in HEK293T cells co-transfected with wild-type (WT) 3 'UTR or mutated-type (MUT) 3'-UTR TNFRSF11a luciferase reporter plasmid and miR-3150b-3p mimics or mimic NC were determined by dual-luciferase reporter assay. (B-C) The mRNA and protein levels of TNFRSF11a in HeLa or SiHa cells with miR-3150b-3p mimic or inhibitor transfection. Data were shown as mean \pm SD. Each experiment was conducted independently for three times. ${ }^{* *} \mathrm{P}<0.01,{ }^{* * *} \mathrm{p}<0.001$ vs mimic/inhibitor NC group.

protein expression. On the contrary, the reduced levels of above-mentioned proteins induced by miR-3150b-3p were remarkably abolished by TNFRSF11a overexpression (online supplementary figure).

\section{DISCUSSION}

Several miRNAs have been demonstrated to be associated with the development and progression of tumorigenesis, including CC. For example, miR-145 inhibited CC cell proliferation in vitro via downregulating fascin actinbundling protein $1 .{ }^{13}$ Yu et al suggested that miR-299-3p repressed CC cell viability and invasive ability via the inhibition of transcription factor $4 .{ }^{14}$ Sun et al demonstrated that miR-889-3p reduced CC cell growth and invasion via targeting fibroblast growth factor receptor $2 .{ }^{15}$ However, the expression and underlying roles of miR-3150b-3p in $\mathrm{CC}$ remain elusive. We suggested that miR-3150b-3p may serve as a tumor suppressor in CC.

Emerging evidence has confirmed that miRNAs could play a certain biological function via binding with specific target genes. ${ }^{16}$ Our findings confirmed that TNFRSF11a was a direct target of miR-3150b-3p that conversely regulated the expression of TNFRSF11a. TNFRSF11a, also known as RANK, is a type- 1 transmembrane protein of 616 amino acids, which plays a vital role in the development and maintenance of diverse tumors. ${ }^{17} 18$ In CC progression,
A

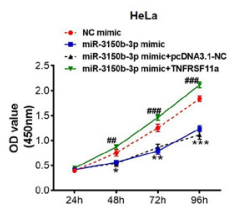

B

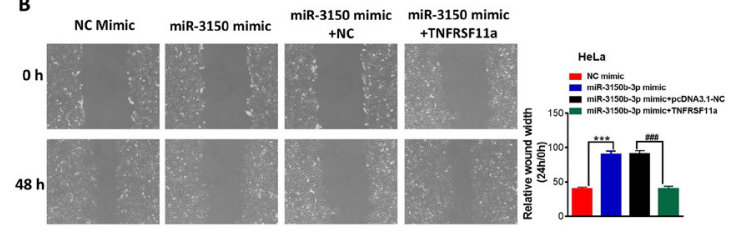

C

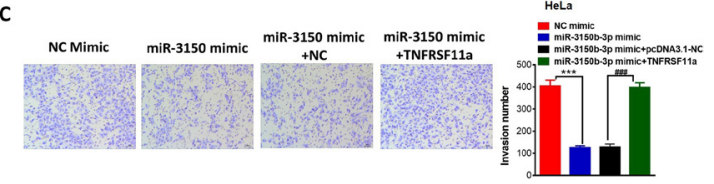

Figure 5 MiR-3150b-3p overexpression alleviated cervical cancer (CC) cell proliferation, migration and invasion via targeting TNFRSF11a. Cell proliferation (A), migration (B) and invasion (C) after transfection of mimic NC or miR-3150b-3p mimic in HeLa cells together with pcDNA3.1 or pcDNA3.1-TNFRSF11a. Data were shown as mean $\pm \mathrm{SD}$. Each experiment was conducted independently for three times. ${ }^{*} P<0.05,{ }^{* *} p<0.01,{ }^{* *} p<0.01$ vs mimic NC group; ${ }^{\# \#} \mathrm{p}<0.01,{ }^{\# \#} \mathrm{p}<0.001$ vs miR-3150b-3p mimic+pcDNA3.1 NC group.

TNFRSF11a promotes CC cell proliferation, migration and invasion, suggesting that anti-TNFRSF11a therapy might be a preventative strategy for $\mathrm{CC}^{8}{ }^{8}$ Rescue assay in this study confirmed suggested that miR-3150b-3p exerted antitumor role in CC via targeting TNFRSF11a.

Several researches have proposed that TNFRSF11a via binding its ligand RANKL, activates various signaling pathways. ${ }^{19}$ In our experiments, we supposed that miR3150b-3p/TNFRSF11a axis exerted its function on CC cells via the inactivation of $\mathrm{p} 38$ MAPK signaling pathways.

Overall, our findings demonstrated that miR-3150b-3p might inhibit CC cell proliferation, migration and invasion by targeting TNFRSF11a via the inactivation of p38 MAPK signaling pathway.

Contributors $Z Y$ conducted most of the experiments and wrote the manuscript; XL conducted the experiments and analyzed the data, LW designed the study and revised the manuscript. All authors have read and approved the manuscript.

Funding The authors have not declared a specific grant for this research from any funding agency in the public, commercial or not-for-profit sectors.

Competing interests None declared.

Patient consent for publication Not required.

Provenance and peer review Not commissioned; externally peer reviewed. Data availability statement Data are available on reasonable request.

Open access This is an open access article distributed in accordance with the Creative Commons Attribution Non Commercial (CC BY-NC 4.0) license, which permits others to distribute, remix, adapt, build upon this work noncommercially, and license their derivative works on different terms, provided the original work is properly cited, an indication of whether changes were made, and the use is non-commercial. See: http://creativecommons.org/ licenses/by-nc/4.0/.

\section{ORCID iD}

Liguo Wang http://orcid.org/0000-0002-0021-6317 


\section{REFERENCES}

1 Manaf RA, Ismail S, Cecilia NC. Global burden of cervical cancer: a literature review. International Journal of Public Health \& Clinical Sciences 2017:4:10-18.

2 Gupta S, Maheshwari A, Parab P, et al. Neoadjuvant chemotherapy followed by radical surgery versus concomitant chemotherapy and radiotherapy in patients with stage IB2, IIA, or IIb squamous cervical cancer: a randomized controlled trial. J Clin Oncol 2018;36:1548-55.

3 Barker HE, Paget JTE, Khan AA, et al. The tumour microenvironment after radiotherapy: mechanisms of resistance and recurrence. Nat Rev Cancer 2015;15:409-25.

4 Beristain AG, Narala SR, Di Grappa MA, et al. Homotypic RANK signaling differentially regulates proliferation, motility and cell survival in osteosarcoma and mammary epithelial cells. J Cell Sci 2012;125:943-55.

5 Walsh MC, Choi Y. Biology of the RANKL-RANK-OPG system in immunity, bone, and beyond. Front Immunol 2014;5:511.

6 Papanastasiou AD, Sirinian C, Kalofonos HP. Identification of novel human receptor activator of nuclear factor- $k B$ isoforms generated through alternative splicing: implications in breast cancer cell survival and migration. Breast Cancer Res 2012;14:R112.

7 Shang W-Q, Li H, Liu L-B, et al. RANKL/RANK interaction promotes the growth of cervical cancer cells by strengthening the dialogue between cervical cancer cells and regulation of IL-8 secretion. Oncol Rep 2015;34:3007-16.

$8 \mathrm{Ma} D$, Chang L-Y, Zhao S, et al. Klf5 promotes cervical cancer proliferation, migration and invasion in a manner partly dependent on TNFRSF11a expression. Sci Rep 2017;7:15683.
9 Hendrickson DG, Hogan DJ, McCullough HL, et al. Concordant regulation of translation and mRNA abundance for hundreds of targets of a human microRNA. PLoS Biol 2009; 7:e1000238.

10 Juan C, Hua Q, Ruping Z, et al. miRNA-489 as a biomarker in diagnosis and treatment of cervical cancer. Bratis/ Lek Listy 2018;119:278-83.

11 Chen G, Huang P, Xie J, et al. microRNA-211 suppresses the growth and metastasis of cervical cancer by directly targeting ZEB1. Mol Med Rep 2018; 17:1275-82.

12 Tan D, Zhou C, Han S, et al. Microrna-378 enhances migration and invasion in cervical cancer by directly targeting autophagy-related protein 12. Mol Med Rep 2018;17:6319-26.

$13 \mathrm{Ma} \mathrm{L}$, Li L-L. miR-145 contributes to the progression of cervical carcinoma by directly regulating FSCN1. Cell Transplant 2019;28:1299-305.

14 Yu Y, Zhao J-D, Yang H. MiR-299-3p inhibits proliferation and invasion of cervical cancer cell via targeting Tcf4. Eur Rev Med Pharmacol Sci 2019;23:5621-7.

15 Sun Y, Cheng Y, Zhang Y, et al. MicroRNA-889-3p targets FGFR2 to inhibit cervical cancer cell viability and invasion. Exp Ther Med 2019;18:1440-8.

16 Kabekkodu SP, Shukla V, Varghese VK, et al. Clustered miRNAs and their role in biological functions and diseases. Biol Rev Camb Philos Soc 2018;93:1955-86.

17 Anderson DM, Maraskovsky E, Billingsley WL, et al. A homologue of the TNF receptor and its ligand enhance T-cell growth and dendritic-cell function. Nature 1997;390:175-9.

$18 \mathrm{Sig} / \mathrm{V}$, Jones LP, Penninger JM. RANKL/RANK: from bone loss to the prevention of breast cancer. Open Biol 2016;6:160230.

19 Chen H, Guo T, Wang D, et al. Vaccaria hypaphorine impairs RANKL-induced osteoclastogenesis by inhibition of ERK, p38, JNK and NF- $\mathrm{KB}$ pathway and prevents inflammatory bone loss in mice. Biomed Pharmacother 2018;97:1155-63. 